\title{
Random-matrix theory of mesoscopic fluctuations in conductors and superconductors
}

\author{
C W J Beenakker \\ Instıtuut Lorentz, Unvversity of Leiden, PO Box 9506, 2300 RA Leiden, The Netherlands
}

(Recelved 25 January 1993)

\begin{abstract}
This paper contains a theoretical study of the sample-to-sample fluctuations in transport properties of phase-coherent, diffusive, quasi-one-dimensional systems The main result is a formula for the variance of the fluctuations of an arbitrary linear statistıc on the transmission eigenvalues $[1 \mathrm{e}$, an observable of the form $\left.A=\sum_{n=1}^{N} f\left(T_{n}\right)\right]$ The formula is the analog of the Dyson-Mehta theorem in the statistical theory of energy levels The analysis is based on an existing random-matrix theory for the joint probability distribution of the transmission eigenvalues $T_{n}(n=1,2, \quad, N)$, and holds in the large- $N$ limit The variance of the fluctuations is shown to be undependent of the sample size or degree of disorder and to have a universal $1 / \beta$ dependence on the symmetry parameter $\beta$ of the matrix ensemble It follows that the universality which was established in the theory of "universal conductance fluctuations" is generic for a whole class of transport properties in mesoscopic conductors and superconductors A further implication of the analysis is that the correlations between the transmission eigenvalues are not precisely described by a logarithmic interaction
\end{abstract}

\section{INTRODUCTION}

Universal conductance fluctuations (UCF) is the phenomenon where sample-to-sample fluctuations in the conductance are of order $e^{2} / h$ at zero temperature, independent of the size of the sample or the degree of disorder as long as the conductor remains in the diffusive transport regime (A diffusive conductor is long compared to the mean free path, but short compared to the localization length ) This universality stands out as one of the most remarkable results in mesoscopic physics ${ }^{1}$

The theory of UCF was originally formulated as a diagrammatic perturbation theory by $\mathrm{Al}^{\prime}$ tshuler $^{2}$ and by Lee and Stone ${ }^{3}$ Subsequently, an alternative nonperturbative approach was developed, based on general properties of the statistics of eigenvalues of random matrices (random Hamiltonians, ${ }^{4}$ or random scattering matrices ${ }^{5-9}$ ) The universality of the conductance fluctuations was shown to originate from the universal eigenvalue repulsion in random-matrix ensembles, discovered long ago in nuclear physics ${ }^{10-12}$ The symmetry class of the ensemble manifests itself in the universal dependence of the variance of the conductance on the presence of a magnetic field or spin-orbit scattering Random-matrix theory offers an understanding of UCF which is both fundamental and intuitive

The relationship between the statistics of energy levels measured in nuclear reactions, on the one hand, and the statistics of conductance fluctuations measured in transport experiments, on the other hand, is described in the review article of Stone et $a l^{7}$ In the former problem it is known that fluctuations in the density of eigenvalues of random Hamiltonians are governed by level repulsion, which depends on the symmetry of the Hamiltonian ensemble - but is independent of the mean level density ${ }^{13-16}$ The same holds for the eigenvalues of random scattering matrices (in the so-called circular ensemble of unitary matrices ${ }^{12}$ ) However, this established universality is not directly applicable to the latter problem of the statistics of conductance fluctuations, because of two essential complications

The first is that the transmission coefficients (the quantities which determine the conductance) are not the elgenvalues of the scattering matrix Instead, the transmission coefficients $T_{n}(n=1,2, \quad, N)$ are the eigenvalues of the matrix product $t t^{\dagger}$, where the transmission matrix $t$ is an $N \times N$ submatrix of the $2 N \times 2 N$ scattering matrix of the conductor This first complication was resolved by Muttalıb, Pichard, and Stone ${ }^{6}$ and by Mello, Pereyra, and Kumar, ${ }^{8}$ who (in two different approaches) determined the Jacobian of the transformation from the space of scattering matrices to the space of transmission eigenvalues It turns out that the repulsion of the variables $\lambda_{n} \equiv\left(1-T_{n}\right) / T_{n}$ (being the ratio of reflection and transmission coefficients) takes the same form as the repulsion of energy levels $E_{n}$

The second complication is that the correlation function of the $\lambda_{n}$ 's is not translationally invariant, due to the positivity constraint on $\lambda$ This constraint $\lambda \geq 0$ follows directly from unitarity of the scattering matrix In contrast, the correlation function in the random-matrix theory of energy levels is translationally invariant over the energy range of interest ${ }^{17}$ This second complication was clearly identified by Stone et al,${ }^{7}$ but remained unresolved

Because of this technical complication, the randommatrix theory of quantum transport could not be as well developed as its counterpart in nuclear physics In that field there exists a formula, due to Dyson and Mehta, ${ }^{18}$ which allows one to compute the variance of any linear 
statistic on the energy levels [i.e., an observable $A=$ $\sum_{n} a\left(E_{n}\right)$, with $a$ an arbitrary function of energy]. The Dyson-Mehta formula reads

$$
\operatorname{Var} A=\frac{1}{\beta} \frac{1}{\pi^{2}} \int_{0}^{\infty} d k|a(k)|^{2} k,
$$

where $a(k)=\int_{-\infty}^{\infty} d E e^{i k E} a(E)$ is the Fourier transform of $a(E)$. The symmetry parameter $\beta$ equals 1,2 , or 4 , depending on whether the Hamiltonian ensemble belongs to the orthogonal, unitary, or symplectic symmetry class. ${ }^{12}$ Equation (1.1) shows that (1) the variance is independent of microscopic parameters and (2) the variance has a universal $1 / \beta$ dependence on the symmetry parameter of the ensemble. No such general result exists for the variance of transport properties. The lack of a formula with the same generality as Eq. (1.1) is being felt especially now that mesoscopic fluctuations in transport properties other than the conductance (both in conductors and in superconductors) have become of interest. Examples are the critical-current fluctuations in Josephson junctions, ${ }^{19}$ conductance fluctuations at normal-superconductor interfaces, ${ }^{20}$ and fluctuations in the shot-noise power of metals. ${ }^{21}$

Here we show one can overcome this obstacle towards the establishment of universality in the random-matrix theory of quantum transport. Our main result is the analog of the Dyson-Mehta formula for the variance of a linear statistic $A=\sum_{n} f\left(T_{n}\right)$ on the transmission eigenvalues:

$$
\operatorname{Var} A=\frac{1}{\beta} \frac{1}{\pi^{2}} \int_{0}^{\infty} d k|F(k)|^{2} k \tanh (\pi k) .
$$

The function $F(k)$ is defined in terms of the function $f(T)$ by the transform

$$
F(k)=\int_{-\infty}^{\infty} d x e^{i k x} f\left(\frac{1}{1+e^{x}}\right) .
$$

The formula (1.2) demonstrates that the universality which was the hallmark of UCF is generic for a whole class of transport properties, viz., those which are linear statistics on the transmission eigenvalues. This generality was anticipated by Imry, ${ }^{5}$ but could not previously be established.

The outline of this paper is as follows. In Sec. II we formulate the problem and summarize the results of the random-matrix theory of Muttalib, Pichard, and Stone, ${ }^{6}$ on which our analysis is based. We mention the limitations inherent in this approach, to which we will return later on in the paper (Sec. VIII). We do not discuss the alternative approaches of Al'tshuler and Shklovskir. ${ }^{4}{ }^{4}$ and of Mello, Pereyra, and Kumar, ${ }^{8}$ to which our method is not applicable. Our method of calculation employs a functional derivative technique, which we introduce in Sec. II. In Sec. III we present an integral equation for the mean density of transmission eigenvalues, in the limit that the dimension $N$ of the transmission matrix goes to infinity. The derivation of this equation, along the lines of an analogous derivation by Dyson, ${ }^{14}$ is given in Appendix A. The solution of the integral equation by Mellin transformation is described in Sec. IV. The solving kernel, combined with the functional derivative relation of Sec. II, directly yields the density-density correlation function of the transmission eigenvalues in the large- $N$ limit. In Sec. V we combine the results of the previous sections to obtain the analog of the Dyson-Mehta formula for the quantum transport problem (see also Appendix B). This formula is applied to a variety of transport properties, in conductors and superconductors, in Sec. VI. In Sec. VII we show that our results agree with the (numerically calculated) large- $N$ limit of a special exactly solvable model (the Laguerre ensemble ${ }^{7,22}$ ). A comparison with other theories of mesoscopic fluctuations is given in Sec. VIII and Appendix C. We conclude in Sec. IX. A brief account of our results has been given in Ref. 23.

\section{FORMULATION OF THE PROBLEM}

We consider a disordered conductor of length $L$ and width $W$ at zero temperature. The elastic scattering of noninteracting electrons at the Fermi level is described by the unitary scattering matrix ( $s$ matrix)

$$
s=\left(\begin{array}{ll}
r_{11} & t_{12} \\
t_{21} & r_{22}
\end{array}\right)
$$

The reflection and transmission matrices $r$ and $t$ are $N \times N$ matrices, $N$ being the number of propagating modes at the Fermi energy. The matrix product $t_{12} t_{12}^{\dagger}$ is Hermitian, and hence has real eigenvalues $T_{n}$ $(n=1,2, \ldots, N)$. Since $t_{12} t_{12}^{\dagger}=r_{11} t_{21}^{\dagger} t_{21} r_{11}^{-1}$ (as follows from unitarity of $s$ ), the matrices $t_{12} t_{12}^{\dagger}$ and $t_{21} t_{21}^{\dagger}$ have the same set of eigenvalues. We refer to the $T_{n}$ 's as the transmission eigenvalues. Unitarity of $s$ also implies that $0 \leq T_{n} \leq 1$ for all $n$. We will study transport properties $A$ of the form

$$
A=\sum_{n=1}^{N} f\left(T_{n}\right) .
$$

A quantity of the form (2.2) is called a linear statistic on the transmission eigenvalues. The word "linear" indicates that $A$ does not contain products of different eigenvalues, but the function $f(T)$ may well depend nonlinearly on $T$.

Starting point of our analysis is the joint probability distribution of transmission eigenvalues obtained in the random-matrix theory of quantum transport. ${ }^{6,7}$ To make contact with that theory we adopt the parametrization

$$
T_{n} \equiv \frac{1}{1+\lambda_{n}}, \quad 0 \leq \lambda<\infty,
$$

and work with a linear statistic on the $\lambda$ 's,

$$
A=\sum_{n=1}^{N} a\left(\lambda_{n}\right) .
$$

Since there is a simple one-to-one relationship between $\lambda$ and $T$, we will still refer to the $\lambda$ 's as "transmission eigenvalues." The distribution of the $\lambda$ 's is given by ${ }^{6,7}$ 


$$
\begin{aligned}
& P\left(\left\{\lambda_{n}\right\}\right)=Z^{-1} \exp \left[-\beta \mathcal{H}\left(\left\{\lambda_{n}\right\}\right)\right], \\
& \mathcal{H}\left(\left\{\lambda_{n}\right\}\right)=-\sum_{\imath<\jmath} \ln \left|\lambda_{\imath}-\lambda_{\jmath}\right|+\sum_{\imath} V\left(\lambda_{\imath}\right),
\end{aligned}
$$

where $Z$ is such that $P$ is normalized to unity,

$$
Z=\int_{0}^{\infty} d \lambda_{1} \cdots \int_{0}^{\infty} d \lambda_{N} \exp \left[-\beta \mathcal{H}\left(\left\{\lambda_{n}\right\}\right)\right]
$$

The parameter $\beta$ depends on the symmetry properties of the ensemble of scattering matrices. If time-reversal symmetry is broken (by a magnetic field), $\beta=2$. In the presence of time-reversal symmetry, $\beta=1$ if the scattering is spin independent, while $\beta=4$ for strong spin-orbit scattering. ${ }^{24,25}$ These three universality classes are referred to as the orthogonal $(\beta=1)$, unitary $(\beta=2)$, and symplectic $(\beta=4)$ ensembles. ${ }^{12}$

The probability distribution (2.5) has the form of a Gibbs distribution, with the symmetry parameter $\beta$ playing the role of inverse temperature, and the "Hamiltonian" $\mathcal{H}$ containing a logarithmic repulsive interaction plus a confining potential $V$. The function $V(\lambda)$ is chosen such that $P$ yields the required average eigenvalue density (which depends on the sample size and the degree of disorder). Note that $V$ may also be a function of $\beta$. The logarithmic interaction has a fundamental geometric origin: The factor $\exp \left(\beta \sum_{2<j} \ln \left|\lambda_{2}-\lambda_{\jmath}\right|\right)=\prod_{2<j}\left|\lambda_{2}-\lambda_{\jmath}\right|^{\beta}$ is the Jacobian associated with the transformation from the space of scattering matrices $s$ to the smaller space of transmission eigenvalues $T_{n} \cdot{ }^{6-9,26}$ The form (2.5) for the probability distribution is based on (a) an isotropy assumption, which implies that flux incident in one scattering channel is, on average, equally distributed among all outgoing channels; and (b) a maximum entropy hypothesis, which yields (2.5) as the least restrictive distribution consistent with a given average eigenvalue density. Assumption (a) requires a conductor much longer than wide, i.e., the quasi-one-dimensional limit $L \gg W$. Furthermore, the conductor should be long compared to the mean free path $l$ for elastic impurity scattering, in order to exclude ballistic transmission. Assumption (b) has been justified by comparison with numerical simulations, ${ }^{6,7,27}$ but there exists no rigorous proof. Indeed, it is conceivable that the true eigenvalue distribution $P\left(\left\{\lambda_{n}\right\}\right)$ cannot be fully described by a one-body potential $V(\lambda)$ plus Jacobian, as in Eq. (2.5), but that it contains additional many-body potentials. These would modify the logarithmic interaction of the $\lambda$ 's. We emphasize this because one of the implications of our analysis will be that Eq. (2.5) is not rigorously valid - although the error is quite small.

The goal of our analysis is to obtain the variance of the linear statistic (2.4) from the eigenvalue distribution function (2.5). To this end we need to know how pairs of transmission eigenvalues are correlated. Our approach is to relate the correlation function to a functional derivative of the eigenvalue density with respect to $V$, and then to evaluate this functional derivative in the limit $N \rightarrow \infty$. This limit, taken at constant $L$ and $l$, ensures that the conductor is short compared to the localization length $\mathrm{Nl}$ - so that it is in the diffusive transport regime, as required for UCF. ${ }^{28}$ In this section we deal with the first step of our program, which is an exercise in statistical mechanics. A similar line of reasoning was used by Politzer, ${ }^{29}$ for a different purpose (viz., to show that $A$ has a Gaussian distribution).

The mean density of transmission eigenvalues $\langle\rho(\lambda)\rangle$ is defined as the ensemble average of the microscopic density $\rho(\lambda)$ :

$$
\begin{aligned}
\rho(\lambda) & =\sum_{n=1}^{N} \delta\left(\lambda-\lambda_{n}\right), \\
\langle\rho(\lambda)\rangle & =\frac{\int d \lambda_{1} \cdots \int d \lambda_{N} \rho(\lambda) \exp (-\beta \mathcal{H})}{\int d \lambda_{1} \cdots \int d \lambda_{N} \exp (-\beta \mathcal{H})} .
\end{aligned}
$$

We define the "two-point correlation function" $K_{2}\left(\lambda, \lambda^{\prime}\right)$ by

$$
K_{2}\left(\lambda, \lambda^{\prime}\right)=\langle\rho(\lambda)\rangle\left\langle\rho\left(\lambda^{\prime}\right)\right\rangle-\left\langle\rho(\lambda) \rho\left(\lambda^{\prime}\right)\right\rangle .
$$

It is related to the "two-level cluster function" $T_{2}\left(\lambda, \lambda^{\prime}\right)$ of Ref. 12 by

$$
K_{2}\left(\lambda, \lambda^{\prime}\right)=T_{2}\left(\lambda, \lambda^{\prime}\right)-\langle\rho(\lambda)\rangle \delta\left(\lambda-\lambda^{\prime}\right) .
$$

We include the singular self-correlation in the correlation function because it contributes to the variance of a linear statistic (see below).

To obtain the required relationship, we take the functional derivative of $\langle\rho(\lambda)\rangle$ with respect to $V\left(\lambda^{\prime}\right)$. Since $\delta \mathcal{H} / \delta V(\lambda)=\rho(\lambda)$, differentiation of Eq. (2.8) yields

$$
\begin{aligned}
\frac{\delta\langle\rho(\lambda)\rangle}{\delta V\left(\lambda^{\prime}\right)}= & -\beta \frac{\int d \lambda_{1} \cdots \int d \lambda_{N} \rho(\lambda) \rho\left(\lambda^{\prime}\right) \exp (-\beta \mathcal{H})}{\int d \lambda_{1} \cdots \int d \lambda_{N} \exp (-\beta \mathcal{H})} \\
& +\beta\left(\frac{\int d \lambda_{1} \cdots \int d \lambda_{N} \rho(\lambda) \exp (-\beta \mathcal{H})}{\int d \lambda_{1} \cdots \int d \lambda_{N} \exp (-\beta \mathcal{H})}\right)\left(\frac{\int d \lambda_{1} \cdots \int d \lambda_{N} \rho\left(\lambda^{\prime}\right) \exp (-\beta \mathcal{H})}{\int d \lambda_{1} \cdots \int d \lambda_{N} \exp (-\beta \mathcal{H})}\right) \\
= & -\beta\left\langle\rho(\lambda) \rho\left(\lambda^{\prime}\right)\right\rangle+\beta\langle\rho(\lambda)\rangle\left\langle\rho\left(\lambda^{\prime}\right)\right\rangle .
\end{aligned}
$$


Hence we obtain the key relation

$$
K_{2}\left(\lambda, \lambda^{\prime}\right)=\frac{1}{\beta} \frac{\delta\langle\rho(\lambda)\rangle}{\delta V\left(\lambda^{\prime}\right)} .
$$

The linear statistic (2.4) can be written in terms of the microscopic eigenvalue density (2.7),

$$
A=\int_{0}^{\infty} d \lambda a(\lambda) \rho(\lambda)
$$

The ensemble average $\langle A\rangle$ is

$$
\langle A\rangle=\int_{0}^{\infty} d \lambda a(\lambda)\langle\rho(\lambda)\rangle,
$$

so that the variance $\operatorname{Var} A \equiv\left\langle A^{2}\right\rangle-\langle A\rangle^{2}$ becomes

$$
\begin{aligned}
\operatorname{Var} A & =-\int_{0}^{\infty} d \lambda \int_{0}^{\infty} d \lambda^{\prime} a(\lambda) a\left(\lambda^{\prime}\right) K_{2}\left(\lambda, \lambda^{\prime}\right) \\
& =-\frac{1}{\beta} \int_{0}^{\infty} d \lambda \int_{0}^{\infty} d \lambda^{\prime} a(\lambda) a\left(\lambda^{\prime}\right) \frac{\delta\langle\rho(\lambda)\rangle}{\delta V\left(\lambda^{\prime}\right)} .
\end{aligned}
$$

This relationship between the variance of a linear statistic and the functional derivative of the density of transmission eigenvalues is an exact consequence of the probability distribution (2.5).

An immediate implication of Eq. (2.15) is that $\operatorname{Var} A \propto$ $1 / \beta$, provided the functional derivative $\delta\langle\rho\rangle / \delta V$ is independent of the symmetry parameter $\beta$. As we will see in the next section, this is indeed the case for $N \rightarrow \infty-$ regardless of any $\beta$ dependence of $V$. Furthermore, since all microscopic details of the system enter via the "potential" $V(\lambda)$, universality of the fluctuations is obtained if $\langle\rho\rangle$ is a linear functional of $V$. Again, this holds for $N \rightarrow \infty$, as we will see next.

\section{INTEGRAL EQUATION FOR THE EIGENVALUE DENSITY}

To evaluate the functional derivative (2.12) we must know how the density of transmission eigenvalues $\langle\rho\rangle$ depends on the potential $V$ in the Hamiltonian (2.5). This problem has been addressed before in the random-matrix theory of energy levels, which is also based on the distribution function (2.5), but without the positivity constraint on $\lambda$. For that case, Dyson ${ }^{14}$ has derived the following equation:

$$
\int d \lambda^{\prime}\left\langle\rho\left(\lambda^{\prime}\right)\right\rangle \ln \left|\lambda-\lambda^{\prime}\right|+\frac{\beta-2}{2 \beta} \ln \langle\rho(\lambda)\rangle=V(\lambda)+\text { const },
$$

where the additive constant is to be determined from the normalization condition

$$
\int d \lambda\langle\rho(\lambda)\rangle=N
$$

The second term on the left-hand side of Eq. (3.1) is of order $N^{-1} \ln N$ relative to the first, and terms of still higher order in $1 / N$ are neglected. To calculate the twopoint correlation function (2.12) in leading order it is sufficient to retain only the first term, so that we can work with the linear integral equation

$$
\int d \lambda^{\prime}\left\langle\rho\left(\lambda^{\prime}\right)\right\rangle \ln \left|\lambda-\lambda^{\prime}\right|=V(\lambda)+\text { const. }
$$

Equation (3.3) has the intuitive "mean-field" interpretation (originally due to Wigner ${ }^{30}$ ) that the "charge density" $\langle\rho\rangle$ adjusts itself to the "external potential" $V$ in such a way that the total force on any charge $\lambda$ vanishes. The more accurate equation (3.1) shows that, in fact, Eq. (3.3) is the leading term in a $1 / N$ expansion.

In Dyson's derivation of Eq. (3.1), essential use is made of the fact that all integrals run from $-\infty$ to $+\infty .{ }^{31}$ In our case, the integration range is from 0 to $\infty$. In Appendix A we show how Dyson's analysis can be modified to incorporate the positivity constraint on $\lambda$. The final result is still Eq. (3.1), i.e., the positivity constraint introduces no extra terms to the order considered.

To obtain the two-point correlation function $K_{2}\left(\lambda, \lambda^{\prime}\right)$ in the limit $N \rightarrow \infty$ we thus need to study the integral equation (3.3). The functional derivative $\delta\langle\rho\rangle / \delta V$ equals the solving kernel of

$$
\int_{0}^{\infty} d \lambda^{\prime} \psi\left(\lambda^{\prime}\right) \ln \left|\lambda-\lambda^{\prime}\right|=\phi(\lambda)+\text { const }
$$

where the additive constant has to be chosen such that $\psi$ has zero mean,

$$
\int_{0}^{\infty} d \lambda \psi(\lambda)=0
$$

since the variations in $\langle\rho\rangle$ have to occur at constant $N$. Because of Eq. (2.12), the integral solution

$$
\psi(\lambda)=\int_{0}^{\infty} d \lambda^{\prime} \beta K_{2}\left(\lambda, \lambda^{\prime}\right) \phi\left(\lambda^{\prime}\right)
$$

of Eq. (3.4) directly determines the two-point correlation function, and hence the variance (2.15) of a linear statistic. Since the integral equation (3.4) does not contain any microscopic parameters and is independent of the symmetry parameter $\beta$, the two statements of universality made at the end of Sec. II are now validated: $\operatorname{Var} A$ is inversely proportional to $\beta$ and is independent of $\mathrm{mi}$ croscopic parameters. To calculate the value of $\operatorname{Var} A$ we have to determine the solving kernel of Eq. (3.4). This is the subject of the next section.

\section{SOLUTION OF THE INTEGRAL EQUATION}

The integral equation (3.4) can be solved analytically by a Mellin-transform technique. We define

$$
\begin{aligned}
x & =\ln \lambda, \quad-\infty<x<\infty, \\
\tilde{\psi}(x) & =e^{x} \psi\left(e^{x}\right), \\
\tilde{\phi}(x) & =\phi\left(e^{x}\right) \\
\tilde{K}_{2}\left(x, x^{\prime}\right) & =e^{x+x^{\prime}} K_{2}\left(e^{x}, e^{x^{\prime}}\right) .
\end{aligned}
$$

In the new variable $x$ Eqs. (3.4) and (3.5) become

$$
\int_{-\infty}^{\infty} d x^{\prime} \tilde{\psi}\left(x^{\prime}\right) C\left(x, x^{\prime}\right)=\tilde{\phi}(x)+\text { const }
$$




$$
\int_{-\infty}^{\infty} d x \tilde{\psi}(x)=0
$$

with kernel $C\left(x, x^{\prime}\right)=\ln \left|e^{x}-e^{x^{\prime}}\right|$. The inverse of $C$ equals $\beta \tilde{K}_{2}$,

$$
\tilde{\psi}(x)=\int_{-\infty}^{\infty} d x^{\prime} \beta \tilde{K}_{2}\left(x, x^{\prime}\right) \tilde{\phi}\left(x^{\prime}\right)
$$

in accordance with Eq. (3.6). To proceed, we note that the integral equation (4.5) is invariant under the transformation

$$
C\left(x, x^{\prime}\right) \rightarrow C\left(x, x^{\prime}\right)+f(x)+g\left(x^{\prime}\right),
$$

since the integral $\int d x^{\prime} \tilde{\psi}\left(x^{\prime}\right) f(x)$ vanishes by virtue of condition (4.6), while the integral $\int d x^{\prime} \tilde{\psi}\left(x^{\prime}\right) g\left(x^{\prime}\right)$ contributes an $x$-independent constant which can be absorbed in the constant at the right-hand side of Eq. (4.5). We now choose $f(x)=-\frac{1}{2} x$ and $g\left(x^{\prime}\right)=-\frac{1}{2} x^{\prime}$. The transformed kernel becomes translationally invariant,

$$
\ln \left|e^{x}-e^{x^{\prime}}\right|-\frac{1}{2}\left(x+x^{\prime}\right)=\ln \left|2 \sinh \left(\frac{x-x^{\prime}}{2}\right)\right| .
$$

In this way we succeeded in reducing the integral equation (3.4) to a convolution,

$$
\int_{-\infty}^{\infty} d x^{\prime} \tilde{\psi}\left(x^{\prime}\right) \ln \left|2 \sinh \left(\frac{x-x^{\prime}}{2}\right)\right|=\tilde{\phi}(x)+\text { const }
$$

which can be solved easily by Fourier transformation.

We define the Fourier transform of an arbitrary function $f(x)$ by

$$
f(k)=\int_{-\infty}^{\infty} d x e^{i k x} f(x) .
$$

Fourier transformation of the kernel yields

$$
\int_{-\infty}^{\infty} d x e^{i k x} \ln \left|2 \sinh \frac{x}{2}\right|=-\frac{\pi}{k} \operatorname{cotanh}(\pi k) .
$$

Hence Eq. (4.10) has the $k$-space solution

$$
\tilde{\psi}(k)=-\frac{k}{\pi} \tanh (\pi k) \tilde{\phi}(k) .
$$

Equation (4.6) is automatically satisfied, provided $\lim _{k \rightarrow 0} k^{2} \tilde{\phi}(k)=0$.

We conclude from Eqs. (4.7) and (4.10) that the twopoint correlation function in the variable $x$ is translationally invariant, $\tilde{K}_{2}\left(x, x^{\prime}\right) \equiv \tilde{K}_{2}\left(x-x^{\prime}\right)$, with Fourier transform

$\tilde{K}_{2}(k) \equiv \int_{-\infty}^{\infty} d x e^{i k x} \tilde{K}_{2}(x)=-\frac{k}{\pi \beta} \tanh (\pi k)$.

Inversion of the Fourier transform yields

$$
\begin{aligned}
\tilde{K}_{2}(x) & \equiv \frac{1}{2 \pi} \int_{-\infty}^{\infty} d k e^{-\imath k x} \tilde{K}_{2}(k) \\
& =-\frac{1}{\pi^{2} \beta} \frac{d^{2}}{d x^{2}} \ln \left|\tanh \frac{x}{4}\right| .
\end{aligned}
$$

In contrast, the two-point correlation function $K_{2}\left(\lambda, \lambda^{\prime}\right)$ in the original variable $\lambda$ is not translationally invariant. Using the relation (4.4) between $\tilde{K}_{2}$ and $K_{2}$, we find from Eq. (4.15) the expression

$$
K_{2}\left(\lambda, \lambda^{\prime}\right)=\frac{1}{\pi^{2} \beta} \frac{\partial}{\partial \lambda} \frac{\partial}{\partial \lambda^{\prime}} \ln \left|\frac{\sqrt{\lambda}-\sqrt{\lambda^{\prime}}}{\sqrt{\lambda}+\sqrt{\lambda^{\prime}}}\right| .
$$

The kernel $K_{2}\left(\lambda, \lambda^{\prime}\right)$ has an integrable singularity for $\lambda=$ $\lambda^{\prime}$. The nonsingular part is obtained by carrying out the differentiation in Eq. (4.16), with the result

$$
K_{2}\left(\lambda, \lambda^{\prime}\right)=\frac{1}{2 \pi^{2} \beta}\left(\lambda \lambda^{\prime}\right)^{-1 / 2}\left(\lambda+\lambda^{\prime}\right)\left(\lambda-\lambda^{\prime}\right)^{-2}
$$

$$
\text { if } \lambda \neq \lambda^{\prime} \text {. }
$$

It is of interest to compare our asymptotic result for the two-point correlation furr ion with the exact result of Slevin, Pichard, and $\mathrm{Mello}^{22}$ for the Laguerre ensemble, defined by Eq. (2.5) with $\beta=2$ and $V(\lambda)=\frac{1}{2} \lambda-\frac{1}{2} \alpha \ln \lambda$. The parameter $\alpha>-1$ is arbitrary (the case $\alpha=0$ has also been considered in Ref. 7). For this ensemble the correlation function can be calculated exactly, using the method of orthogonal polynomials. ${ }^{12}$ Slevin, Pichard, and Mello find for the two-level cluster function $T_{2}\left(\lambda, \lambda^{\prime}\right)$ the formula

$$
\begin{aligned}
T_{2}\left(\lambda, \lambda^{\prime}\right)= & \left(\lambda \lambda^{\prime}\right)^{\alpha} e^{-\lambda-\lambda^{\prime}} \\
& \times\left\{\sum_{n=0}^{N-1} \frac{\Gamma(n+1)}{\Gamma(n+\alpha+1)} L_{n}^{\alpha}(\lambda) L_{n}^{\alpha}\left(\lambda^{\prime}\right)\right\}^{2} .
\end{aligned}
$$

For $N^{-1 / 2} \ll \lambda, \lambda^{\prime} \ll N^{1 / 2}$ an asymptotic expansion of the Laguerre polynomials $L_{n}^{\alpha}$ yields ${ }^{22}$

$$
\begin{aligned}
T_{2}\left(\lambda, \lambda^{\prime}\right)= & \frac{1}{4 \pi^{2}}\left(\lambda \lambda^{\prime}\right)^{-1 / 2} \\
& \times\left\{\frac{\sin \left[2 N^{1 / 2}\left(\sqrt{\lambda}-\sqrt{\lambda^{\prime}}\right)\right]}{\sqrt{\lambda}-\sqrt{\lambda^{\prime}}}\right. \\
& \left.-\frac{\cos \left[2 N^{1 / 2}\left(\sqrt{\lambda}+\sqrt{\lambda^{\prime}}\right)-\pi \alpha\right]}{\sqrt{\lambda}+\sqrt{\lambda^{\prime}}}\right\}^{2} .
\end{aligned}
$$

If $\lambda \neq \lambda^{\prime}$ the two-level cluster function $T_{2}\left(\lambda, \lambda^{\prime}\right)$ and the two-point correlation function $K_{2}\left(\lambda, \lambda^{\prime}\right)$ are identical [cf. Eq. (2.10)], so that we can compare with Eq. (4.17). The sine and cosine in Eq. (4.19) oscillate rapidly for $N \rightarrow \infty$, and hence may be smoothed out by averaging $\lambda$ and $\lambda^{\prime}$ over a range $\delta \lambda$ much larger than $1 / N$, but much smaller than 1. The $\sin ^{2}$ and $\cos ^{2}$ terms average to $\frac{1}{2}$, while the cross term $\sin \times \cos$ averages to zero, independently of $\alpha$. Equation (4.19) then reduces to

$$
\begin{aligned}
T_{2}\left(\lambda, \lambda^{\prime}\right)= & \frac{1}{4 \pi^{2}}\left(\lambda \lambda^{\prime}\right)^{-1 / 2}\left\{\frac{1 / 2}{\left(\sqrt{\lambda}-\sqrt{\lambda^{\prime}}\right)^{2}}\right. \\
& \left.+\frac{1 / 2}{\left(\sqrt{\lambda}+\sqrt{\lambda^{\prime}}\right)^{2}}\right\} \\
= & \frac{1}{4 \pi^{2}}\left(\lambda \lambda^{\prime}\right)^{-1 / 2}\left(\lambda+\lambda^{\prime}\right)\left(\lambda-\lambda^{\prime}\right)^{-2},
\end{aligned}
$$


which is the same as our Eq. (4.17) for $\beta=2$.

For $N \rightarrow \infty$ the peak in $T_{2}\left(\lambda, \lambda^{\prime}\right)$ at $\lambda \approx \lambda^{\prime}$ can be approximated by the delta function $\langle\rho(\lambda)\rangle \delta\left(\lambda-\lambda^{\prime}\right)$. [Note that $\int d \lambda^{\prime} T_{2}\left(\lambda, \lambda^{\prime}\right)=\langle\rho(\lambda)\rangle$, by definition.] It is not obvious analytically that the remaining singularity in $K_{2}\left(\lambda, \lambda^{\prime}\right) \equiv T_{2}\left(\lambda, \lambda^{\prime}\right)-\langle\rho(\lambda)\rangle \delta\left(\lambda-\lambda^{\prime}\right)$ has the asymptotic form (4.16). A numerical demonstration of the equivalence will be given in Sec. VII.

\section{FORMULA FOR THE VARIANCE OF A LINEAR STATISTIC}

We are now ready to evaluate the variance of the linear statistic $A=\sum_{n} a\left(\lambda_{n}\right)$. We define

$$
\tilde{a}(x)=a\left(e^{x}\right) .
$$

Using also the definition (4.4), Eq. (2.15) takes the form

$$
\operatorname{Var} A=-\int_{-\infty}^{\infty} d x \int_{-\infty}^{\infty} d x^{\prime} \tilde{a}(x) \tilde{a}\left(x^{\prime}\right) \tilde{K}_{2}\left(x, x^{\prime}\right)
$$

In the preceding section we have found that the two-point correlation function $\tilde{K}_{2}\left(x, x^{\prime}\right)$ is translationally invariant, with Fourier transform $\tilde{K}_{2}(k)$ given by Eq. (4.14). We define the Fourier transform of $\tilde{a}(x)$ according to Eq. (4.11),

$$
\tilde{a}(k)=\int_{-\infty}^{\infty} d x e^{\imath k x} \tilde{a}(x)=\int_{0}^{\infty} d \lambda \lambda^{i k-1} a(\lambda) .
$$

The Fourier transform with respect to $x$ is a Mellin transform with respect to $\lambda$. Equation (5.2) becomes in $k$ space

$$
\operatorname{Var} A=-\frac{1}{2 \pi} \int_{-\infty}^{\infty} d k|\tilde{a}(k)|^{2} \tilde{K}_{2}(k) .
$$

Substituting Eq. (4.14) we obtain the formula

$$
\operatorname{Var} A=\frac{1}{\beta} \frac{1}{2 \pi^{2}} \int_{-\infty}^{\infty} d k|\tilde{a}(k)|^{2} k \tanh (\pi k)
$$

for the variance of a linear statistic on the transmission eigenvalues. Equation (5.5) is equivalent to Eq. (1.2) in the Introduction.

The formula (5.5) is for the quantum transport problem what the Dyson-Mehta formula was for the problem of the statistics of energy levels. A derivation of the latter formula along the lines set out in the present paper (which is more general than previous derivations) is given in Appendix B.

Before proceeding to the application of Eq. (5.5) to a variety of linear statistics, we give for completeness the $\lambda$ representation of this formula. Substituting Eq. (4.16) into Eq. (2.15), and carrying out two partial integrations, one finds

$$
\begin{aligned}
\operatorname{Var} A=-\frac{1}{\beta} \frac{1}{\pi^{2}} \int_{0}^{\infty} d \lambda \int_{0}^{\infty} & d \lambda^{\prime}\left(\frac{d a(\lambda)}{d \lambda}\right)\left(\frac{d a\left(\lambda^{\prime}\right)}{d \lambda^{\prime}}\right) \\
& \times \ln \left|\frac{\sqrt{\lambda}-\sqrt{\lambda^{\prime}}}{\sqrt{\lambda}+\sqrt{\lambda^{\prime}}}\right| \cdot \quad(5.6)
\end{aligned}
$$

Equation (5.6) requires that $a(\lambda)$ is differentiable. In par- ticular, $\operatorname{Var} A$ diverges logarithmically for a step function, $a(\lambda)=\theta\left(\lambda_{c}-\lambda\right)$. For such artificial linear statistics the variance does not have a universal $N \rightarrow \infty$ limit, but increases as $\ln N$ for large $N .{ }^{18}$ All physical properties considered in the next section, however, are smooth (differentiable) functions of $\lambda$.

\section{APPLICATIONS}

We list various applications of the variance formula (5.5) without discussion, which we defer to Sec. VIII.

\section{A. Conductance}

The conductance $G$ is related to the transmission eigenvalues by the Landauer formula ${ }^{1}$

$$
G / G_{0}=\sum_{n=1}^{N} T_{n}=\sum_{n=1}^{N} \frac{1}{1+\lambda_{n}} .
$$

Here $G_{0}=2 e^{2} / h$ is the conductance quantum. The conductance is a linear statistic of the form (2.4), with $a(\lambda)=(1+\lambda)^{-1}$. The Mellin transform of $a(\lambda)$, i.e., the Fourier transform of $\left(1+e^{x}\right)^{-1}$, is

$$
\tilde{a}(k)=\int_{-\infty}^{\infty} d x e^{i k x} \frac{1}{1+e^{x}}=-\frac{i \pi}{\sinh (\pi k)} .
$$

Substitution into Eq. (5.5) yields the variance

$$
\operatorname{Var}\left(G / G_{0}\right)=\beta^{-1} \int_{-\infty}^{\infty} d k \frac{k}{\sinh (2 \pi k)}=\frac{1}{8} \beta^{-1} .
$$

\section{B. Shot noise}

The shot-noise power $P$ is given by ${ }^{32}$

$$
P / P_{0}=\sum_{n=1}^{N} T_{n}\left(1-T_{n}\right)=\sum_{n=1}^{N} \frac{\lambda_{n}}{\left(1+\lambda_{n}\right)^{2}},
$$

with $P_{0}=2 e|U| G_{0}$ ( $U$ is the applied voltage). The Mellin transform of $a(\lambda)=\lambda(1+\lambda)^{-2}$ is

$\tilde{a}(k)=\int_{-\infty}^{\infty} d x e^{i k x} \frac{e^{x}}{\left(1+e^{x}\right)^{2}}=\frac{\pi k}{\sinh (\pi k)}$.

Hence the variance becomes

$\operatorname{Var}\left(P / P_{0}\right)=\beta^{-1} \int_{-\infty}^{\infty} d k \frac{k^{3}}{\sinh (2 \pi k)}=\frac{1}{64} \beta^{-1}$.

\section{Normal-superconductor interface}

The conductance $G_{\text {NS }}$ of a disordered microbridge between a normal and a superconducting reservoir is related to the transmission eigenvalues in the normal state by ${ }^{33}$

$G_{\mathrm{NS}} / G_{0}=2 \sum_{n=1}^{N}\left(\frac{T_{n}}{2-T_{n}}\right)^{2}=\sum_{n=1}^{N} \frac{2}{\left(1+2 \lambda_{n}\right)^{2}}$.

This expression holds only in zero magnetic field $(\beta=1)$. 
The Mellin transform of $a(\lambda)=2(1+2 \lambda)^{-2}$ is

$\tilde{a}(k)=\int_{-\infty}^{\infty} d x e^{i k x} \frac{2}{\left(1+2 e^{x}\right)^{2}}=-\frac{2 \pi k+2 \pi i}{\sinh (\pi k)} e^{-i k \ln 2}$,

which leads to the variance

$$
\operatorname{Var}\left(G_{\mathrm{NS}} / G_{0}\right)=4 \int_{-\infty}^{\infty} d k \frac{k^{3}+k}{\sinh (2 \pi k)}=\frac{9}{16}
$$

where we have set $\beta$ equal to 1 .

\section{Josephson junction}

The supercurrent-phase relationship $I(\phi)$ of a pointcontact Josephson junction can be expressed in terms of the normal-state transmission eigenvalues, ${ }^{19}$

$$
I(\phi) / I_{0}=\sum_{n=1}^{N} \frac{\frac{1}{2} T_{n} \sin \phi}{\left[1-T_{n} \sin ^{2}(\phi / 2)\right]^{1 / 2}},
$$

where again $\beta=1$ is required. Here $I_{0}=e \Delta / \hbar$ is the supercurrent quantum ( $\Delta$ being the energy gap in the superconductor). In this case it is easiest to start from the variance formula (5.6) in the $\lambda$ representation, which can be integrated numerically. The resulting root-meansquare value $\operatorname{rms} I \equiv(\operatorname{Var} I)^{1 / 2}$ is plotted in Fig. 1, for phase differences in the interval $(0, \pi)$ (since the variance is $\pi$ periodic). The limiting behavior at the edges of the interval can be computed analytically from Eqs. (5.5) and (6.10). for small $\phi, \operatorname{rms} I(\phi)$ increases linearly from zero with slope $2^{-5 / 2} e \Delta / \hbar$; for $\phi \rightarrow \pi, \operatorname{rms} I(\phi)$ approaches the value $\pi^{-1} e \Delta / \hbar$. The increase is almost, but not quite, monotonic (there is a slight maximum at $\phi \approx 2.7$ ).

For comparison, we have also plotted in Fig. 1 the ensemble average $\langle I(\phi)\rangle$ of the supercurrent, given by ${ }^{34,35}$

$$
\langle I(\phi)\rangle=\frac{\pi \Delta}{e}\langle G\rangle \cos (\phi / 2) \operatorname{arctanh}[\sin (\phi / 2)] .
$$

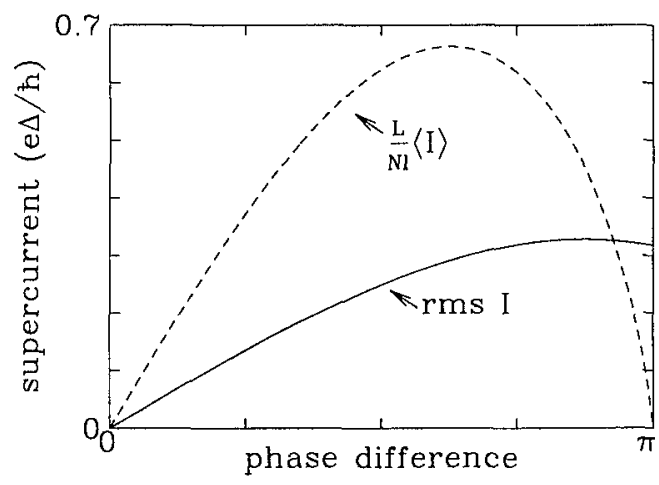

FIG. 1. Supercurrent $I$ (in units of $e \Delta / \hbar$ ), as a function of the phase difference $\phi$ across the Josephson junction. The solid curve is the square root of the variance of $I$, computed from Eqs. (5.6) and (6.10). The dotted curve is the ensemble average of $I$ (scaled by $N l / L$ ), computed from Eq. (6.11). The root-mean-square fluctuation $\operatorname{rms} I_{\mathrm{c}}$ in the critical current equals $\operatorname{rms} I$ at $\phi=1.97$.
Here $\langle G\rangle=\left(2 e^{2} / h\right) N l / L$ is the ensemble-averaged conductance of the same junction (with length $L$ and mean free path $l$ ) in the normal state. For $\phi \rightarrow \pi,\langle I(\phi)\rangle$ goes to zero while $\operatorname{rms} I(\phi)$ remains finite. These two limits can be reconciled by noting that our result for the variance holds in the limit $N \rightarrow \infty$ at constant $\phi$. Taking the limit $N \rightarrow \infty$ before taking the limit $\phi \rightarrow \pi$ ensures that the fluctuations in the supercurrent remain smaller than the average. A calculation to higher order in $1 / N$ is needed to show that $\operatorname{rms} I(\phi)$ goes to zero at $\phi=\pi$.

The maximum value of the supercurrent is known as the critical current of the Josephson junction. The critical current $I_{\mathrm{c}} \equiv \max I(\phi) \equiv I\left(\phi_{\mathrm{c}}\right)$ is not by definition a linear statistic, since the phase difference $\phi_{\mathrm{c}}$ at which the maximum supercurrent is reached depends itself on all the transmission eigenvalues. It is therefore not possible in general to write $I_{c}$ in the form $\sum_{n} f\left(T_{n}\right)$, as required for a linear statistic. However, $I_{\mathrm{c}}$ does become a linear statistic in the limit $N \rightarrow \infty .3{ }^{35}$ To see this, we write

$$
I(\phi)=\langle I(\phi)\rangle[1+\epsilon X(\phi)], \epsilon \equiv L / N l,
$$

where the function $X(\phi)$ accounts for the sample-tosample fluctuations of $I(\phi)$ around the ensemble average $\langle I(\phi)\rangle$. One has $\langle X\rangle=0, \operatorname{rms} X=\mathcal{O}(1)$. We now expand $I_{c}$ to lowest order in $\epsilon$. We define $\phi_{c} \equiv \phi_{c}^{(0)}+\epsilon \phi_{c}^{(1)}$, where $\max \langle I(\phi)\rangle \equiv\left\langle I\left(\phi_{c}^{(0)}\right)\right\rangle$. The phase difference $\phi_{c}^{(0)}=1.97$ is the phase difference at which the ensembleaveraged supercurrent (6.11) reaches its maximum. One has

$$
\begin{aligned}
I_{c} & \equiv\left\langle I\left(\phi_{c}\right)\right\rangle\left[1+\epsilon X\left(\phi_{c}\right)\right] \\
& =\left\langle I\left(\phi_{c}^{(0)}+\epsilon \phi_{c}^{(1)}\right)\right\rangle\left[1+\epsilon X\left(\phi_{c}^{(0)}+\epsilon \phi_{c}^{(1)}\right)\right] \\
& =\left\langle I\left(\phi_{c}^{(0)}\right)\right\rangle\left[1+\epsilon X\left(\phi_{c}^{(0)}\right)+\mathcal{O}\left(\epsilon^{2}\right)\right]=I\left(\phi_{c}^{(0)}\right),
\end{aligned}
$$

up to terms of order $\epsilon^{2}$. In the third equality we have used that, by definition, $d\langle I\rangle / d \phi=0$ at $\phi=\phi_{c}^{(0)}$. Since $I\left(\phi_{c}^{(0)}\right)$ is a linear statistic on $T_{n}$, we conclude that the critical current $I_{c}$ is a linear statistic on the transmission eigenvalues in the limit $\epsilon \equiv L / N l \rightarrow 0$, with $\operatorname{rms} I_{c}=$ $\operatorname{rms} I\left(\phi_{c}^{(0)}\right)$. From the data in Fig. 1 we find

$$
\operatorname{rms} I_{c}=0.29 \mathrm{e \Delta} / \hbar \text {. }
$$

\section{COMPARISON WITH NUMERICAL CALCULATIONS}

As an independent check of the validity of our asymptotic analysis, we have compared Eq. (5.5) with an exactly solvable model. We consider the Laguerre ensemble (cf. Sec. IV), defined by Eq. (2.5) with $\beta=2$ and $V(\lambda)=\frac{1}{2} \lambda-\frac{1}{2} \alpha \ln \lambda$. The parameter $\alpha>-1$ plays the role of a microscopic parameter. In this ensemble, the variance of the linear statistic (2.4) is given exactly by ${ }^{7,22}$

$$
\begin{aligned}
\operatorname{Var} A= & \int_{0}^{\infty} d \lambda a(\lambda)^{2} k_{N, \alpha}(\lambda, \lambda) \\
& -\int_{0}^{\infty} d \lambda \int_{0}^{\infty} d \lambda^{\prime} a(\lambda) a\left(\lambda^{\prime}\right)\left[k_{N, \alpha}\left(\lambda, \lambda^{\prime}\right)\right]^{2},
\end{aligned}
$$




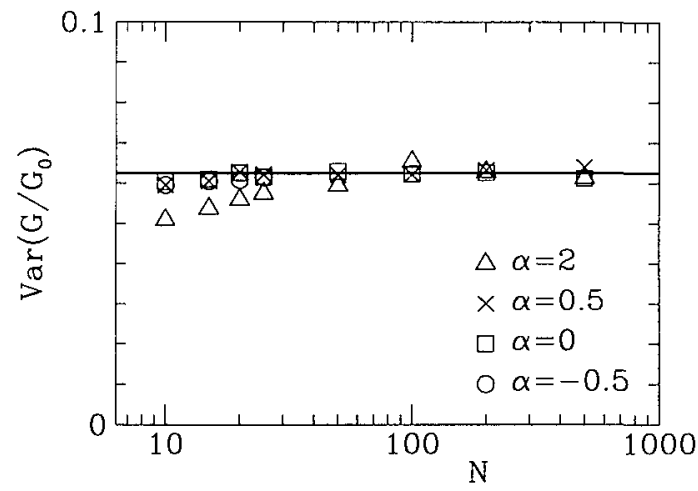

FIG. 2. Variance of the conductance $G$ (in units of $G_{0}=$ $2 e^{2} / h$ ), as a function of the number of channels $N$. The data points are obtained by integration of the exact correlation function in the Laguerre ensemble (Refs. 7 and 22), for various values of the microscopic parameter $\alpha$ [Eqs. (7.1) and (7.2)]. The estimated error in the numerical integration is \pm 0.001 . (For $\alpha=-0.5$ we could only integrate with the required accuracy for $N$ up to 25.) The horizontal line at 0.0625 is the $\alpha$-independent value predicted in the limit $N \rightarrow \infty$ by Eq. (5.5), for $\beta=2$.

$$
\begin{aligned}
k_{N, \alpha}\left(\lambda, \lambda^{\prime}\right)= & \left(\lambda \lambda^{\prime}\right)^{\alpha / 2} e^{-\left(\lambda+\lambda^{\prime}\right) / 2} \\
& \times \sum_{n=0}^{N-1} \frac{\Gamma(n+1)}{\Gamma(n+\alpha+1)} L_{n}^{\alpha}(\lambda) L_{n}^{\alpha}\left(\lambda^{\prime}\right) .
\end{aligned}
$$

We evaluated the integrals over the Laguerre polynomials $L_{n}^{\alpha}$ numerically. In Fig. 2 we show the comparison for the variance of the conductance [Eq. (6.1)]. For the Laguerre ensemble (which has $\beta=2$ ) we would expect from Eq. (6.3) that $\operatorname{Var}\left(G / G_{0}\right)=0.0625$ for $N \gg 1$, independent of $N$ and $\alpha$. As one can see in Fig. 2, this is indeed what we find (within numerical accuracy) from integration of the exact correlation function. Note that the convergence is not uniform in $\alpha$. For $\alpha=0$ the large- $N$ limit is reached already at $N \approx 10$, while for $\alpha=2$ we need to go up to $N \approx 100$.

We have checked for all the transport properties mentioned in Sec. VI that the variances predicted by Eq. (5.5) agree with the numerical results from the Laguerre ensemble for large $N$. We consider this strong evidence for the validity of the asymptotic analysis leading to Eq. (5.5).

\section{COMPARISON WITH OTHER THEORIES}

We return to the applications of the variance formula given in Sec. VI, and compare these with previous theoretical work on mesoscopic fluctuations.

\section{A. Conductance}

The diagrammatic perturbation theory ${ }^{2,3}$ of UCF yields $\operatorname{Var}\left(G / G_{0}\right)=\frac{2}{15} \beta^{-1}$ for a quasi-one-dimensional conductor (i.e., a conductor much longer than it is wide). The coefficient $\frac{1}{8}$ in Eq. (6.3) is close to, but not precisely identical to, $\frac{2}{15}$. The smallness of the difference explains why it was not noticed previously. In particular, the difference is too small to resolve by numerical simulations of a microscopic model. From a practical point of view, the discrepancy is not really significant, but conceptually it has the important implication that the random-matrix theory based on the probability distribution (2.5) is not rigorously equivalent to the diagrammatic perturbation theory of UCF, which we hold to be exact. The conclusion is that the interaction between the $\lambda$ 's is not precisely logarithmic.

A second implication of $\frac{1}{8} \neq \frac{2}{15}$ is that the randommatrix theory based on the probability distribution (2.5) (the so-called "global approach" of Muttalib, Pichard, and Stone ${ }^{6}$ ) is not precisely equivalent to the "local approach" of Mello, Pereyra, and Kumar. ${ }^{8}$ The local approach is based on an evolution equation for the probability distribution as a function of the length of the sample, and yields $\operatorname{Var} G=\frac{2}{15} \beta^{-1}$ in agreement with the diagrammatic perturbation theory. ${ }^{9}$ Previous work by Mello and Pichard ${ }^{36}$ argues for the equivalence of the local and global approaches. As we discuss in Appendix $\mathrm{C}$, their argument is insufficient: It starts from a onebody potential $V(\lambda)$, i.e., it assumes that the interaction between the $\lambda$ 's is precisely logarithmic. We now know that this is an approximation (albeit an excellent one). It would be worthwhile to try to derive the interaction potential from the asymptotic solution of Mello's evolution equation and see how it differs from a two-body logarithmic interaction. We have not made any progress in this direction.

The above discussion of a small deviation from purely logarithmic interaction refers to the quasi-onedimensional transport regime. In higher dimensions the situation is different. We know from the diagrammatic perturbation theory ${ }^{2,3}$ that $\operatorname{Var} G$ depends on the geometry of the conductor. The isotropy assumption restricts the random-matrix theory (both global and local approaches) to the quasi-one-dimensional limit of a long and narrow conductor. It has been conjectured ${ }^{22}$ that the geometry dependence of $\operatorname{Var} G$ can still be described by the probability distribution (2.5), through a dimensionality-dependent confining potential $V(\lambda)$. The variance formula (5.5) demonstrates that this is not the case, since $\operatorname{Var} G$ is independent of $V$ in the large- $N$ limit. The implication is that in higher dimensions the logarithmic interaction in Eq. (2.5) is no longer a suitable approximation. This conclusion was reached independently, through numerical simulations, by Slevin, Pichard, and Muttalib. ${ }^{27}$

\section{B. Shot noise}

A previous calculation of the variance of the shot-noise power based on Mello's evolution equation (the local approach mentioned above) has been carried out by De Jong and the present author. ${ }^{21}$ The calculation applies a moment expansion technique, ${ }^{9}$ which works for linear statistics $A=\sum_{n} f\left(T_{n}\right)$ for which $f(T)$ is a low-order polynomial in $T$. [For the shot-noise $f(T) \propto T(1-T)$; cf. Eq. (6.4).] The result of Ref. 21 is $\operatorname{Var}\left(P / P_{0}\right)=\frac{46}{2835} \beta^{-1}$. 
The coefficient $\frac{1}{64} \approx 0.0156$ in Eq. (6.6) is again close to, but not precisely identical to $\frac{46}{2835} \approx 0.0162$. This is a similar discrepancy to that for the conductance, and similar comments apply.

\section{Normal-superconductor interface}

A calculation of the variance of the conductance of a normal-superconductor (NS) junction was carried out by means of diagrammatic perturbation theory by Takane and Ebisawa. ${ }^{20}$ Only one of the contributing diagrams was evaluated explicitly, from which they estimated $\operatorname{Var} G_{\mathrm{NS}} \approx 6 \operatorname{Var} G$ (where $G$ is the conductance of the junction in the normal state). Numerical simulations by these same authors ${ }^{37}$ yielded the estimate $\operatorname{Var} G_{\mathrm{NS}} \approx 4 \operatorname{Var} G$. From Eqs. (6.3) and (6.9) we find $\operatorname{Var} G_{\mathrm{NS}}=\frac{9}{2} \operatorname{Var} G$. The difference with the numerical simulations is within the numerical accuracy of the latter.

In the presence of a magnetic field the conductance $G_{\text {NS }}$ of the NS junction is no longer a linear statistic. ${ }^{33}$ Numerical simulations ${ }^{38}$ show that the breaking of timereversal symmetry $(\beta=1 \rightarrow \beta=2)$ leaves $\operatorname{Var} G_{\text {NS }}$ essentially unchanged - while the variance $\operatorname{Var} G$ of the normal-state conductance is reduced by a factor of 2 (as expected from the $1 / \beta$ dependence of the variance of a linear statistic). An analytical theory of the nonuniversal $\beta$ dependence of $\operatorname{Var} G_{\mathrm{NS}}$ is still lacking.

\section{Josephson junction}

The order of magnitude $\operatorname{rms} I_{c} \simeq e \Delta / \hbar$ of the fluctuations in the critical current was reported previously by the present author. ${ }^{19,35}$ This result holds for a pointcontact Josephson junction, which is short compared to the superconducting coherence length $\xi=\left(\hbar v_{F} l / \pi \Delta\right)^{1 / 2}$ (where $v_{F}$ is the Fermi velocity and $l$ the mean free path). If the junction is long compared to $\xi$, the supercurrent is no longer a linear statistic on the transmission eigenvalues. ${ }^{19}$ The long-junction limit $L \gg \xi$ was studied by Al'tshuler and Spivak. ${ }^{39}$ They find the nonuniversal result $\operatorname{rms} I_{c} \simeq e v_{F} l / L^{2}$, which depends on sample size and degree of disorder.

\section{CONCLUSION}

We have presented a method to treat the eigenvalue correlations in random-matrix ensembles. The method is based on (1) a functional derivative relation between the mean eigenvalue density and the density-density correlation function and (2) an integral equation ${ }^{14}$ for the eigenvalue density, valid asymptotically in the high-density (large- $N$ ) limit. When applied to the random-matrix theory of energy-level statistics, our method provides a more general derivation of the Dyson-Mehta formula ${ }^{18}$ for the variance of a linear statistic on the energy levels. When applied to the random-matrix theory of quantum transport, the functional-derivative method yields the corresponding formula for the variance of a linear statistic on the transmission eigenvalues. This formula demonstrates that the universality which was established in the theory of universal conductance fluctuations ${ }^{2,3}$ is generic for a whole class of transport properties in conductors and superconductors. Such universality was anticipated ${ }^{5}$ from the random-matrix theory of energy levels, but could not previously be established because of the absence of translational invariance of the correlation function of transmission coefficients (originating from the unitarity of the scattering matrix). ${ }^{7}$ The functional-derivative method presented here requires no translational invariance, and thus allows one to solve this problem. The solution has revealed a small numerical discrepancy with the diagrammatic perturbation theory, which implies that the eigenvalue repulsion is not precisely logarithmic in the ratio of reflection to transmission coefficients (the $\lambda$ variables) - as assumed thus far.

It is likely that the functional-derivative method described in this paper can be of use for other problems in random-matrix theory. One such application is the recent work by Jalabert, Pichard, and the present author on the problem of long-range energy-level interaction in metallic particles. ${ }^{41}$ In this inverse problem the functional-derivative method was used to calculate the deviations from logarithmic energy-level repulsion implied by the known ${ }^{4}$ two-point correlation function of the energy levels. We expect other applications to follow.

\section{ACKNOWLEDGMENTS}

I have benefited from discussions with R. A. Jalabert, M. J. M de Jong, J. M. J. van Leeuwen, I. K. Marmorkos, J.-L. Pichard, B. Rejaei, and A. D. Stone. I thank R. A. Jalabert for helping me with the numerical calculations. This research was supported financially by the "Nederlandse organisatie voor Wetenschappelijk Onderzoek" (NWO) via the "Stichting voor Fundamenteel Onderzoek der Materie" (FOM).

\section{APPENDIX A: DERIVATION OF EQ. (3.1)}

Dyson's derivation ${ }^{14}$ of the integral equation (3.1) for $\langle\rho(\lambda)\rangle$ makes essential use of the fact that the variables $\lambda_{n}$ are free to vary from $-\infty$ to $+\infty$. In the quantum transpo. $t$ problem, however, the $\lambda_{n}$ 's are strictly positive. Here we show how Dyson's derivation can be modified to account for the positivity constraint.

The first step is to transform from the variables $\lambda_{n}$ to the variables $x_{n} \equiv \ln \lambda_{n}$. Since $\lambda_{n} \in(0, \infty), x_{n} \in$ $(-\infty, \infty)$. The probability distribution (2.5) becomes, in the new variables,

$$
\begin{aligned}
\tilde{P}\left(\left\{x_{n}\right\}\right)=Z^{-1} \exp \left[-\beta \tilde{\mathcal{H}}\left(\left\{x_{n}\right\}\right)\right], & \\
\tilde{\mathcal{H}}\left(\left\{x_{n}\right\}\right)=-\sum_{\imath<j} \ln \left|e^{x_{2}}-e^{x_{3}}\right| & +\sum_{\imath} V\left(e^{x_{\imath}}\right) \\
& -\beta^{-1} \sum_{\imath} x_{\imath} .
\end{aligned}
$$

The factor $\exp \left(\sum_{\imath} x_{\imath}\right)=\prod_{\imath} \lambda_{2}$ is the Jacobian for the 
transformation from $\lambda$ to $x$. By using the identity (4.9), the "Hamiltonian" $\tilde{\mathcal{H}}\left(\left\{x_{n}\right\}\right)$ for the $x$ variables can be rewritten in terms of a translationally invariant interaction plus a one-body potential,

$$
\begin{aligned}
& \tilde{\mathcal{H}}\left(\left\{x_{n}\right\}\right)=-\sum_{\imath<J} \ln \left|2 \sinh \left(\frac{x_{\imath}-x_{2}}{2}\right)\right|+\sum_{\imath} \tilde{V}\left(x_{\imath}\right), \\
& \tilde{V}(x)=V\left(e^{x}\right)-\left[\frac{1}{2}(N-1)+\beta^{-1}\right] x
\end{aligned}
$$

After these preliminaries we proceed as follows. The distribution function $\tilde{P}\left(\left\{x_{n}\right\}\right)$ satisfies the differential equations (one for each $i=1,2, \ldots, N$ )

$$
\frac{\partial \tilde{P}}{\partial x_{\imath}}+\beta \tilde{P} \frac{\partial \tilde{\mathcal{H}}}{\partial x_{\imath}}=0
$$

We multiply Eq. (A3) by $\delta\left(x-x_{\imath}\right)$, integrate over $x_{1}, x_{2}, \ldots, x_{N}$, and sum over $i$. The result is

$$
\begin{aligned}
\frac{d}{d x}\langle\tilde{\rho}(x)\rangle+\beta \int_{-\infty}^{\infty} d x_{1} \cdots \int_{-\infty}^{\infty} & d x_{N} \tilde{P}\left(\left\{x_{n}\right\}\right) \\
& \times \sum_{\imath=1}^{N} \delta\left(x-x_{\imath}\right) \frac{\partial \tilde{\mathcal{H}}}{\partial x_{\imath}}=0
\end{aligned}
$$

where $\langle\tilde{\rho}(x)\rangle$ is the mean density of the $x_{n}$ 's,

$$
\begin{aligned}
\langle\tilde{\rho}(x)\rangle & =\left\langle\sum_{\imath=1}^{N} \delta\left(x-x_{\imath}\right)\right\rangle \\
& \equiv \int_{-\infty}^{\infty} d x_{1} \cdots \int_{-\infty}^{\infty} d x_{N} \tilde{P}\left(\left\{x_{n}\right\}\right) \sum_{\imath=1}^{N} \delta\left(x-x_{\imath}\right) .
\end{aligned}
$$

Substitution of the expression (A2) for $\tilde{\mathcal{H}}$ into Eq. (A4) leads to

$$
\begin{aligned}
& \frac{d}{d x}\langle\tilde{\rho}(x)\rangle+\beta\langle\tilde{\rho}(x)\rangle \frac{d}{d x} \tilde{V}(x) \\
& \quad-\frac{1}{2} \beta \mathrm{P} \int_{-\infty}^{\infty} d x^{\prime}\left\langle\tilde{\rho}_{2}\left(x, x^{\prime}\right)\right\rangle \operatorname{cotanh}\left(\frac{x-x^{\prime}}{2}\right)=0,
\end{aligned}
$$

where $\mathrm{P} \int$ indicates the principal value of the integral. The pair density $\left\langle\tilde{\rho}_{2}\left(x, x^{\prime}\right)\right\rangle$ is defined by

$$
\left\langle\tilde{\rho}_{2}\left(x, x^{\prime}\right)\right\rangle=\left\langle\sum_{\imath \neq \jmath} \delta\left(x-x_{\imath}\right) \delta\left(x^{\prime}-x_{\jmath}\right)\right\rangle .
$$

The pair density is symmetric in its arguments, $\left\langle\tilde{\rho}_{2}\left(x, x^{\prime}\right)\right\rangle=\left\langle\tilde{\rho}_{2}\left(x^{\prime}, x\right)\right\rangle$, and satisfies the normalization

$$
\int_{-\infty}^{\infty} d x^{\prime}\left\langle\tilde{\rho}_{2}\left(x, x^{\prime}\right)\right\rangle=(N-1)\langle\tilde{\rho}(x)\rangle .
$$

Following Ref. 14 we decompose the pair density into a correlated and an uncorrelated part,

$$
\left\langle\tilde{\rho}_{2}\left(x, x^{\prime}\right)\right\rangle=\langle\tilde{\rho}(x)\rangle\left\langle\tilde{\rho}\left(x^{\prime}\right)\right\rangle\left[1-y\left(x, x^{\prime}\right)\right] .
$$

The function $y\left(x, x^{\prime}\right)=y\left(x^{\prime}, x\right)$ is symmetric, and satisfies

$$
\int_{-\infty}^{\infty} d x^{\prime} y\left(x, x^{\prime}\right)\left\langle\tilde{\rho}\left(x^{\prime}\right)\right\rangle=1
$$

in view of the normalization (A8). Substitution of the definition (A9) into Eq. (A6) leads to

$$
\begin{aligned}
\frac{d}{d x}\langle\tilde{\rho}(x)\rangle+\beta\langle\tilde{\rho}(x)\rangle \frac{d}{d x}[\tilde{V}(x) & +U(x)] \\
& +\frac{1}{2} \beta\langle\tilde{\rho}(x)\rangle I(x)=0,
\end{aligned}
$$

with the definitions

$$
\begin{aligned}
& I(x)=\mathrm{P} \int_{-\infty}^{\infty} d x^{\prime}\left\langle\tilde{\rho}\left(x^{\prime}\right)\right\rangle y\left(x, x^{\prime}\right) \operatorname{cotanh}\left(\frac{x-x^{\prime}}{2}\right), \\
& U(x)=-\int_{-\infty}^{\infty} d x^{\prime}\left\langle\tilde{\rho}\left(x^{\prime}\right)\right\rangle \ln \left|2 \sinh \left(\frac{x-x^{\prime}}{2}\right)\right| .
\end{aligned}
$$

Equation (A11) is still exact. To introduce the approximation we need one further piece of notation. We reexpress the function $y\left(x, x^{\prime}\right)$ in terms of the sum and difference variables $t=\frac{1}{2}\left(x+x^{\prime}\right)$ and $s=x^{\prime}-x$ :

$$
y\left(x, x^{\prime}\right)=Y\left[\frac{1}{2}\left(x+x^{\prime}\right), x^{\prime}-x\right] \equiv Y(t, s) .
$$

The function $Y(t, s)=Y(t,-s)$ is even in $s$. The normalization (A10) becomes

$$
\int_{-\infty}^{\infty} d s Y\left(x+\frac{1}{2} s, s\right)\langle\tilde{\rho}(x+s)\rangle=1 .
$$

Similarly, the integral (A12) takes the form

$$
I(x)=-\mathrm{P} \int_{-\infty}^{\infty} d s Y\left(x+\frac{1}{2} s, s\right)\langle\tilde{\rho}(x+s)\rangle \operatorname{cotanh}\left(\frac{1}{2} s\right) .
$$

By substituting the Taylor expansions

$$
\begin{aligned}
& Y\left(x+\frac{1}{2} s, s\right)=Y(x, s)+\frac{1}{2} s \frac{\partial}{\partial x} Y(x, s)+\cdots, \\
& \langle\tilde{\rho}(x+s)\rangle=\langle\tilde{\rho}(x)\rangle+s \frac{d}{d x}\langle\tilde{\rho}(x)\rangle+\cdots, \\
& \operatorname{coth}\left(\frac{1}{2} s\right)=2 s^{-1}+\frac{1}{6} s+\cdots
\end{aligned}
$$

into Eq. (A16), we obtain an expansion of $I(x)$ in higher and higher moments $Y_{p}(x)$ of $Y(x, s)$ with respect to $s$,

$$
Y_{p}(x)=\int_{-\infty}^{\infty} d s Y(x, s) s^{p} .
$$

Because of the symmetry $Y(t, s)=Y(t,-s)$ only even moments contribute $\left[Y_{p}(x) \equiv 0\right.$ for $p$ odd]. Following Dyson, ${ }^{14}$ we neglect the second and higher moments. An order of magnitude estimate suggests that the error involved in neglecting $Y_{p}$ for $p \geq 2$ is of order $N^{-2}$. Dyson argues that the error is actually of order $N^{-2} \ln N$, by comparison with exact results for the distribution of the spacing of eigenvalues.

Since $Y_{-1}(x)$ and $Y_{1}(x)$ are identically zero, only $Y_{0}(x)$ contributes to $I(x)$ to second order. Substitution of the 
Taylor expansions (A17)-(A19) into Eq. (A16) yields

$$
I(x)=-\langle\tilde{\rho}(x)\rangle \frac{d}{d x} Y_{0}(x)-2 Y_{0}(x) \frac{d}{d x}\langle\tilde{\rho}(x)\rangle .
$$

Similarly, substitution of the Taylor expansions into Eq. (A15) yields

$$
\langle\tilde{\rho}(x)\rangle Y_{0}(x)=1 .
$$

Combining Eqs. (A21) and (A22) we find

$$
I(x)=-\langle\tilde{\rho}(x)\rangle^{-1} \frac{d}{d x}\langle\tilde{\rho}(x)\rangle .
$$

Hence Eq. (A11) takes the form

$$
\left(1-\frac{1}{2} \beta\right) \frac{d}{d x}\langle\tilde{\rho}(x)\rangle+\beta\langle\tilde{\rho}(x)\rangle \frac{d}{d x}[\tilde{V}(x)+U(x)]=0,
$$

or equivalently [using definitions (A2) and (A13)]

$$
\begin{array}{r}
\int_{-\infty}^{\infty} d x^{\prime}\left\langle\tilde{\rho}\left(x^{\prime}\right)\right\rangle \ln \left|2 \sinh \left(\frac{x-x^{\prime}}{2}\right)\right|+\frac{\beta-2}{2 \beta} \ln \langle\tilde{\rho}(x)\rangle \\
=V\left(e^{x}\right)-\frac{N}{2} x+\frac{\beta-2}{2 \beta} x+\text { const. }
\end{array}
$$

The final step of our derivation is to transform back from $x$ to $\lambda=e^{x}$. The densities are related by $\langle\tilde{\rho}(x)\rangle d x=$ $\langle\rho(\lambda)\rangle d \lambda$, hence

$$
\langle\tilde{\rho}(x)\rangle=\lambda\langle\rho(\lambda)\rangle .
$$

Using also the identity (4.9), plus the normalization $\int\langle\rho\rangle d \lambda=N$, we find from Eq. (A25) the result

$$
\int_{0}^{\infty} d \lambda^{\prime}\left\langle\rho\left(\lambda^{\prime}\right)\right\rangle \ln \left|\lambda-\lambda^{\prime}\right|+\frac{\beta-2}{2 \beta} \ln \langle\rho(\lambda)\rangle=V(\lambda)+\text { const. }
$$

This is the integral equation (3.1).

\section{APPENDIX B: DYSON-MEHTA FORMULA REVISITED}

The functional-derivative method developed in this paper for the quantum transport problem can be equally well applied to the statistics of energy levels. In that problem, we recall, the variable $\lambda$ is free to vary from $-\infty$ to $+\infty$, and hence we can define a Fourier transform with respect to $\lambda$ :

$$
a(k)=\int_{-\infty}^{\infty} d \lambda e^{\imath k \lambda} a(\lambda) .
$$

The integral equation (3.3) is now a convolution (since the integral ranges over the whole real axis). Fourier transformation of the kernel $\int_{-\infty}^{\infty} d \lambda e^{\imath k \lambda} \ln |\lambda|=-\pi /|k|$ yields the functional derivative

$$
\begin{aligned}
\frac{\delta\langle p(\lambda)\rangle}{\delta V\left(\lambda^{\prime}\right)} & =\frac{1}{2 \pi} \int_{-\infty}^{\infty} d k \exp \left[i k\left(\lambda-\lambda^{\prime}\right)\right](-\pi /|k|)^{-1} \\
& =-\frac{1}{\pi^{2}} \frac{d^{2}}{d\left(\lambda-\lambda^{\prime}\right)^{2}} \ln \left|\lambda-\lambda^{\prime}\right|
\end{aligned}
$$

Using Eq. (2.12) we find that the (translationally invariant) two-point correlation function $K_{2}\left(\lambda, \lambda^{\prime}\right) \equiv K_{2}(\lambda-$ $\left.\lambda^{\prime}\right)$ is given by

$$
K_{2}(\lambda)=-\frac{1}{\pi^{2} \beta} \frac{d^{2}}{d \lambda^{2}} \ln |\lambda| .
$$

The Fourier-transformed correlation function $K_{2}(k)=$ $-|k| / \pi \beta$ is simply $1 / \beta$ times the inverse of the Fouriertransformed interaction kernel. Substitution of the twopoint correlation function into Eq. (2.15) yields the variance of the linear statistic (2.4),

$$
\operatorname{Var} A=\frac{1}{\beta} \frac{1}{2 \pi^{2}} \int_{-\infty}^{\infty} d k|a(k)|^{2}|k| .
$$

This is the Dyson-Mehta formula quoted in the Introduction.

The difference between Eqs. (5.5) and (B4) originates from the positivity constraint on $\lambda$ in the quantum transport problem. Dyson and Mehta ${ }^{18}$ derived their formula by approximating an exact expression for the two-point correlation function in the Gaussian unitary ensemble of random Hamiltonians [defined by Eq. (2.5) with $\beta=2$ and $V(\lambda) \propto \lambda^{2}$ ]. A recent treatment by Mehta ${ }^{40}$ deals with the Gaussian orthogonal and Gaussian symplectic ensembles $(\beta=1$ and $\beta=4)$, still requiring a quadratic $V(\lambda)$. The present derivation is more general, showing that Eq. (B4) holds for any $\beta$ and $V$.

\section{APPENDIX C: GLOBAL VERSUS LOCAL APPROACH}

The present paper is based on the probability distribution function (2.5) for the transmission eigenvalues. This is the so-called global approach to random-matrix theory. ${ }^{6}$ The so-called local approach starts from the evolution equation ${ }^{8}$

$$
\begin{aligned}
l \frac{\partial}{\partial L} P\left(\left\{\lambda_{n}\right\}\right)= & \frac{2}{\beta N+2-\beta} \\
& \times \sum_{\imath=1}^{N} \frac{\partial}{\partial \lambda_{\imath}}\left(\lambda_{\imath}\left(1+\lambda_{\imath}\right) J\left(\left\{\lambda_{n}\right\}\right)\right. \\
& \left.\times \frac{\partial}{\partial \lambda_{2}} \frac{P\left(\left\{\lambda_{n}\right\}\right)}{J\left(\left\{\lambda_{n}\right\}\right)}\right),
\end{aligned}
$$

which governs the sample-length dependence of the probability distribution in the quasi-one-dimensional limit. Here $J\left(\left\{\lambda_{n}\right\}\right)$ denotes the Jacobian,

$$
J\left(\left\{\lambda_{n}\right\}\right) \equiv \prod_{\imath<\jmath}\left|\lambda_{\imath}-\lambda_{\jmath}\right|^{\beta},
$$

associated with the transformation from matrix to eigenvalue space. It was believed until now that the global and local approaches were equivalent, i.e., that they would give the same large- $N$ results for the mesoscopic fluctuations if the potential $V(\lambda)$ in Eq. (2.5) was suitably chosen. ${ }^{7,36}$ The present theory has shown that this is not correct: The variance of the conductance is 
$\operatorname{Var}\left(G / G_{0}\right)=\frac{1}{8} \beta^{-1}$ in the global approach, independent of $V(\lambda)$, whereas the local approach is known ${ }^{9}$ to yield $\operatorname{Var}\left(G / G_{0}\right)=\frac{2}{15} \beta^{-1}$. In Sec. VIII we have discussed the implications of this difference for the interaction of the eigenvalues. In this appendix we reconsider the argument of Mello and Pichard ${ }^{36}$ for the equivalence of the two theories, and indicate why their argument is insufficient.

Consider the probability distribution function

$$
\begin{aligned}
& P\left(\left\{\lambda_{n}\right\}\right)=Z^{-1} \exp \left[-\beta \mathcal{H}\left(\left\{\lambda_{n}\right\}\right)\right], \\
& \mathcal{H}\left(\left\{\lambda_{n}\right\}\right)=-\sum_{2<\jmath} \ln \left|\lambda_{2}-\lambda_{3}\right|+V\left(\left\{\lambda_{n}\right\}\right),
\end{aligned}
$$

which is more general than Eq. (2.5) because $V$ is now allowed to be a many-body potential. By construction, the function $P\left(\left\{\lambda_{n}\right\}\right)$ satisfies

$$
\frac{\partial P}{\partial \lambda_{\imath}}+\beta P \frac{\partial \mathcal{H}}{\partial \lambda_{\imath}}=0, \quad i=1,2, \ldots, N
$$

By integrating Eq. (C4) one readily shows that the mean density of eigenvalues

$$
\begin{aligned}
\langle\rho(\lambda)\rangle & =\left\langle\sum_{\imath=1}^{N} \delta\left(\lambda-\lambda_{\imath}\right)\right\rangle \\
& \equiv \int_{0}^{\infty} d \lambda_{1} \cdots \int_{0}^{\infty} d \lambda_{N} P\left(\left\{\lambda_{n}\right\}\right) \sum_{\imath=1}^{N} \delta\left(\lambda-\lambda_{\imath}\right)
\end{aligned}
$$

satisfies the relation

$\beta^{-1} \frac{d}{d \lambda}\langle\rho(\lambda)\rangle-\mathrm{P} \int_{0}^{\infty} d \lambda^{\prime} \frac{\left\langle\rho_{2}\left(\lambda, \lambda^{\prime}\right)\right\rangle}{\lambda-\lambda^{\prime}}=\langle f(\lambda)\rangle$,

where $\mathrm{P}$ indicates the principal value. The function $\left\langle\rho_{2}\left(\lambda, \lambda^{\prime}\right)\right\rangle$ is the pair density of eigenvalues,

$$
\left\langle\rho_{2}\left(\lambda, \lambda^{\prime}\right)\right\rangle=\left\langle\sum_{\imath \neq j} \delta\left(\lambda-\lambda_{2}\right) \delta\left(\lambda^{\prime}-\lambda_{\jmath}\right)\right\rangle,
$$

and $\langle f(\lambda)\rangle$ is the mean force density due to the potential $V$,

$$
\langle f(\lambda)\rangle=-\left\langle\sum_{\imath} \delta\left(\lambda-\lambda_{\imath}\right) \frac{\partial}{\partial \lambda_{\imath}} V\left(\left\{\lambda_{n}\right\}\right)\right\rangle .
$$

Following Ref. 36, let us now assume that the probability distribution $P$ defined in Eq. (C4) evolves with sample length according to the evolution equation (C1) of the local approach. By substituting Eq. (C4) into Eq. (C1), and integrating, one obtains for the eigenvalue density the evolution equation

$l \frac{\partial}{\partial L}\langle\rho(\lambda)\rangle=\frac{2 \beta}{\beta N+2-\beta} \frac{\partial}{\partial \lambda}(\lambda(1+\lambda)\langle f(\lambda)\rangle)$.

On the other hand, direct integration of the evolution equation $(\mathrm{C} 1)$ yields

$$
\begin{aligned}
l \frac{\partial}{\partial L}\langle\rho(\lambda)\rangle= & \frac{2 \beta}{\beta N+2-\beta} \frac{\partial}{\partial \lambda} \\
& \times\left(\lambda ( 1 + \lambda ) \left[\beta^{-1} \frac{\partial}{\partial \lambda}\langle\rho(\lambda)\rangle\right.\right. \\
& \left.\left.\quad-\mathrm{P} \int_{0}^{\infty} d \lambda^{\prime} \frac{\left\langle\rho_{2}\left(\lambda, \lambda^{\prime}\right)\right\rangle}{\lambda-\lambda^{\prime}}\right]\right) .
\end{aligned}
$$

(C10)

The two equations (C9) and (C10) are consistent because of the relation (C6).

In the global approach the potential $V$ is assumed to be a one-body potential, i.e., $V\left(\left\{\lambda_{n}\right\}\right)=\sum_{2} V\left(\lambda_{2}\right)$. The force density $\langle f(\lambda)\rangle$ is then equal to $-\langle\rho(\lambda)\rangle d V(\lambda) / d \lambda$. Equation (C6) becomes for large $N$ the integral equation (3.1) (cf. Appendix A), which ensures that Eqs. (C9) and (C10) are consistent. In this way Mello and Pichard ${ }^{36}$ were able to demonstrate the consistency of the global and local approach, assuming that $V$ is a one-body potential. However, as demonstrated above, the consistency holds for any many-body potential $V\left(\left\{\lambda_{n}\right\}\right)$ - so that this argument by itself is insufficient to decide whether $V$ is a one-body potential or not. We now know that it is not.
${ }^{\mathrm{I}}$ Two recent reviews are Mesoscopic Phenomena in Solnds, edited by B. L. Al'tshuler, P. A. Lee, and R. A. Webb (North-Holland, Amsterdam, 1991) and C. W. J. Beenakker and H. van Houten, Solid State Phys. 44, 1 (1991).

${ }^{2}$ B. L. Al'tshuler, Pis'ma Zh. Eksp. Teor. Fiz. 41, 530 (1985) [JETP Lett. 41, 648 (1985)].

${ }^{3}$ P. A. Lee and A. D. Stone, Phys. Rev. Lett. 55, 1622 (1985); P. A. Lee, A. D. Stone, and H. Fukuyama, Phys. Rev. B 35, 1039 (1987).

${ }^{4}$ B. L. Al'tshuler and B. I. Shklovskir, Zh. Eksp. Teor. Fiz. 91, 220 (1986) [Sov. Phys. JETP 64, 127 (1986)].

${ }^{5}$ Y. Imry, Europhys. Lett. 1, 249 (1986).

${ }^{6}$ K. A. Muttalib, J.-L. Pichard, and A. D. Stone, Phys. Rev. Lett. 59, 2475 (1987).

${ }^{7}$ A. D. Stone, P. A. Mello, K. A. Muttalib, and J.-L. Pichard, in Mesoscopıc Phenomena in Solids, edited by B. L. Al'tshuler, P. A. Lee, and R. A. Webb (North-Holland, Amsterdam, 1991).

${ }^{8}$ P. A. Mello, P. Pereyra, and N. Kumar, Ann. Phys. (N.Y.)
181, 290 (1988).

${ }^{9}$ P. A. Mello, Phys. Rev. Lett. 60, 1089 (1988); P. A. Mello and A. D. Stone, Phys. Rev. B 44, 3559 (1991).

${ }^{10}$ E. P. Wigner, SIAM Rev. 9, 1 (1967).

${ }^{11}$ T. A. Brody, J. Flores, J. B. French, P. A. Mello, A. Pandey, and S. S. M. Wong, Rev. Mod. Phys. 53, 385 (1981).

${ }^{12}$ M. L. Mehta, Random Matruces, 2nd ed. (Academic, New York, 1991).

${ }^{13}$ D. Fox and P. B. Kahn, Phys. Rev. (N.Y.) 134, B1151 (1964).

${ }^{14}$ F. J. Dyson, J. Math. Phys. 13, 90 (1972).

${ }^{15}$ A. Pandey, Ann. Phys. (N.Y.) 134, 110 (1981).

${ }^{16}$ R. D. Kamien, H. D. Politzer, and M. B. Wise, Phys. Rev. Lett. 60, 1995 (1988).

${ }^{17}$ One might object that the eigenvalues $E_{n}$ in the Hamiltonian ensemble are also bounded from below, just as the variables $\lambda_{n}$. The difference is that the quantities of interest in the Hamiltonian ensemble are sensitive only to energy levels in the bulk of the spectrum, where the presence of a 
lower bound on the E's is irrelevant. Transport properties, in contrast, are sensitive to transmission coefficients close to unity, i.e., to $\lambda$ 's close to the lower bound at $\lambda=0$. In the quantum transport problem it is the bulk of the spectrum of the $\lambda$ 's which is irrelevant. Note, however, that if for some reason one would like to develop a statistical theory of energy levels close to the ground state, one could directly apply the results of this paper for that purpose.

${ }^{18}$ F. J. Dyson and M. L. Mehta, J. Math. Phys. 4, 701 (1963).

${ }^{19}$ C. W. J. Beenakker, Phys. Rev. Lett. 67, 3836 (1991); 68, 1442(E) (1992).

${ }^{20}$ Y. Takane and H. Ebisawa, J. Phys. Soc. Jpn. 60, 3130 (1991).

${ }^{21}$ M. J. M. de Jong and C. W. J. Beenakker, Phys. Rev. B 46, 13400 (1992).

${ }^{22}$ K. Slevin, J.-L. Pichard, and P. A. Mello, Europhys. Lett. 16, 649 (1991).

${ }^{23}$ C. W. J. Beenakker, Phys. Rev, Lett. 70, 1155 (1993).

${ }^{24}$ N. Zanon and J.-L. Pichard, J. Phys. (Paris) 49, 907 (1988).

${ }^{25}$ In the symplectic ensemble $(\beta=4)$, the transmission matrix has dimension $2 N$, rather than $N$, to accommodate the spin-flip scattering. The transmission eigenvalues, however, are twofold degenerate (Kramers degeneracy, see Ref. 24), and hence there are only $N$ independent eigenvalues. In the linear statistics (2.2) and (2.4) the sum over $n$ is intended to run only over these independent transmission eigenvalues.

${ }^{26}$ The analysis in Refs. 6-9 starts from the space of transfer matrices rather than from the space of scattering matrices. The Jacobian for the transformation to transmission eigenvalues is the same in the two cases.
${ }^{27}$ K. Slevin, J.-L. Pichard, and K. A. Muttalib, J. Phys. (Paris) (to be published).

${ }^{28}$ Throughout this paper we use the limit $N \rightarrow \infty$ to indicate the diffusive transport regime $N \gg L / l \gg 1$.

${ }^{29}$ H. D. Politzer, Phys. Rev. B 40, 11917 (1989).

${ }^{30}$ E. P. Wigner, in Statistical Theories of Spectra: Fluctuations, edited by C. E. Porter (Academic, New York, 1965).

${ }^{31}$ The precise point at which the positivity constraint invalidates Dyson's derivation of our Eq. (3.1) is Eq. (3.18) of Ref. 14.

${ }^{32}$ M. Büttiker, Phys. Rev. Lett. 65, 2901 (1990).

${ }^{33}$ C. W. J. Beenakker, Phys. Rev. B 46, 12841 (1992).

${ }^{34}$ I. O. Kulik and A. N. Omel'yanchuk, Pis'ma Zh. Eksp. Teor. Fiz. 21, 216 (1975) [JETP Lett. 21, 96 (1975)].

${ }^{35} \mathrm{C}$. W. J. Beenakker, in Transport Phenomena in Mesoscopic Systems, edited by H. Fukuyama and T. Ando (Springer, Berlin, 1992).

${ }^{36}$ P. A. Mello and J.-L. Pichard, Phys. Rev. B 40, 5276 (1989).

${ }^{37}$ Y. Takane and H. Ebisawa, J. Phys. Soc. Jpn. 61, 2858 (1992).

${ }^{38}$ I. K. Marmorkos, C. W. J. Beenakker, and R. A. Jalabert (unpublished).

${ }^{39}$ B. L. Al'tshuler and B. Z. Spivak, Zh. Eksp. Teor. Fiz. 92, 609 (1987) [Sov. Phys. JETP 65, 343 (1987)].

${ }^{40}$ See Ref. 12, Appendix A.39. The factor $2 / \beta \pi^{2}$ in Eq. (A.39.9) should read $1 / \beta \pi^{2}$.

${ }^{41}$ R. A. Jalabert, J.-L. Pichard, and C. W. J. Beenakker (unpublished). 